\title{
Trabectedin in combination with pegylated liposomal doxorubicin in patients with ovarian tumors
}

\author{
ALESSANDRO VILLANUCCI ${ }^{1}$, KETTY TAVELLA ${ }^{1}$, LAURA VANNINI ${ }^{2}$, VIRGINIA ROSSI $^{2}$, \\ STEFANIA NOBILI $^{2}$, GIANNI AMUNNI ${ }^{2}$, TERESITA MAZZEI ${ }^{2}$ and ENRICO MINI ${ }^{2}$ \\ ${ }^{1}$ Department of Oncology, Hospital-University Agency of Careggi; \\ ${ }^{2}$ Department of Oncology, University of Florence, I-50134 Florence, Italy
}

Received January 31, 2018; Accepted September 6, 2018

DOI: $10.3892 /$ mco.2018.1729

\begin{abstract}
The majority of patients with ovarian cancer will experience relapse and thus require second-line therapy. While platinum-based therapies are the primary treatments for refractory disease other options are required, particularly for those with partially platinum-sensitive disease as their response rates are lower. Agents that can resensitize relapsed ovarian cancers to platinum, including trabectedin, are therefore of increasing interest. Trabectedin is a multitarget agent that has a complex, novel mechanism of action and has exhibited promising results in platinum-sensitive ovarian cancer when in combination with pegylated liposomal doxorubicin (PLD). The present study conducted retrospective analysis involving 11 cases (median age 60 years; range 45-75 years) of recurrent ovarian tumors and partial platinum sensitivity undergoing treatment with trabectedin + PLD. The cohort consisted of 7 serous carcinomas, 1 endometrial carcinoma, 2 undifferentiated carcinomas, and 1 mucinous carcinoma. Of the 11 patients, 4 exhibited a complete response, 3 achieved stable disease, and 4 had progression of disease. Mean overall survival was 32.42 months and median progression-free survival was 5.9 months. Trabectedin in combination with PLD was well tolerated in terms of gastrointestinal and hematological toxicity; Grade 3 cutaneous toxicity and grade 3 neutropenia were each observed in $18.2 \%$ of patients. There were no grade 4 events. Thus, the present study supports the use of trabectedin + PLD in patients with relapsed ovarian cancer and partial platinum sensitivity, with predictable and manageable toxicity.
\end{abstract}

Correspondence to: Dr Alessandro Villanucci, Department of Oncology, Hospital-University Agency of Careggi, Largo Brambilla 3, I-50134 Florence, Italy

E-mail: villanuccia@aou-careggi.toscana.it

Abbreviations: PFI, platinum-free interval; NER, nucleotide excision repair; PLD, pegylated liposomal doxorubicin

Key words: trabectedin, pegylated liposomal doxorubicin, ovarian cancer, recurrence, treatment, survival, retrospective analysis

\section{Introduction}

In patients with ovarian cancer, at least $80 \%$ will experience relapse and will require second-line therapy. Additional treatment of these patients is currently complex, but the length of the platinum-free interval (PFI) is an important clinical consideration (1). Since the effectiveness of platinum retreatment is dependent on the relapse-free and treatment-free intervals, stratification of patients based on PFI can help clinicians to decide the optimal therapeutic strategy. At present, according to the PFI, patients are normally classified as those with fully platinum sensitive disease (PFI $>12$ months), partially platinum sensitive disease (PFI 6-12 months), platinum resistant disease (PFI $<6$ months) and refractory disease (progression during the last line of platinum therapy or within 4 weeks of the last platinum dose) (2). Recently, there has been some discussion that the patients should no longer to be categorized using the PFI (with an arbitrary cut-off of 6-months), but rather 'according to the question: Is platinum still an option for the patient?' (3).

Notwithstanding, in recurrent ovarian tumors, the PFI remains a primary prognostic factor for both progression-free survival (PFS) and overall survival (OS) $(4,5)$. Nonetheless, it must be considered that PFI is in reality a continuous variable that does not always adequately reflect disease prognosis. Patients with a fully platinum-sensitive relapse typically receive a second-line salvage therapy based on retreatment with platinum-containing regimens with response rates that vary from 30 to $75 \%$, while patients with partially platinum-sensitive recurrent disease typically have lower response rates when retreated with platinum (27-33\%) (6). Moreover, from 20 to $40 \%$ of all ovarian cancer patients will have partially platinum-sensitive disease for whom the optimal treatment sequence is still controversial (6). Indeed, among those with platinum sensitive disease, patients with partially platinum sensitive disease are the most challenging to clinically manage. Given this, there is thus intense interest in providing new therapeutic options for these patients. Agents that may resensitize tumors to platinum have been generating interest (7), and among these trabectedin is being investigated in relapsed ovarian cancer.

Trabectedinis a multitarget agent that was originally extracted from a species of marine tunicate, Ecteinascidia turbinate, and has a complex, novel mechanism of action. Firstly, trabectedin 
has been shown to bind to the minor groove of double-stranded DNA, causing double-strand breaks $(8,9)$. Secondly, trabectedin appears to affect the cell cycle by causing apoptosis of cancer cells and by downregulating transcription factors associated with cell proliferation (9). Due to its cytotoxic effects on tumor-associated macrophages, trabectedin may further inhibit the release of cytokines by monocytes and macrophages in the tumor microenvironment (10). Importantly, trabectedin has also been reported to interact directly with components involved in nucleotide excision repair (NER), thereby inhibiting repair of specific NER substrates and forming a ternary complex that appears to be associated with cell death (7). Since the NER pathway is responsible for repairing platinum-DNA adducts in cellular DNA, NER aberrations will increase the sensitivity of tumors to platinum. This novel aspect of trabectedin is thus related to the high-sensitivity seen in NER-proficient cells upon exposure to platinum, which are generally more resistant to platinum compounds. These findings thus provide a clear rationale for the benefits of sequential treatment with trabectedin and platinum compounds (7).

First approved in the EU as monotherapy for treatment of advanced soft tissue sarcoma in 2007, trabectedin is also indicated for the treatment of patients with relapsed, platinum-sensitive ovarian cancer in combination with pegylated liposomal doxorubicin (PLD) since 2009.

In ovarian tumors, trabectedin in combination with PLD has best been studied in the OVA-301 trial (11). In fact, among the various options investigated, early studies with trabectedin in combination with PLD suggested that the combination was associated with a significant advantage in survival compared to PLD alone (11). In the entire population, median PFS was 7.3 months with trabectedin + PLD vs. 5.8 months with PLD (HR, 0.79; $\mathrm{P}=0.0190)$. In the partially platinum-sensitive subpopulation, trabectedin + PLD led to a $41 \%$ decrease in the risk of death ( $\mathrm{HR}, 0.59 ; \mathrm{P}=0.0015)$, with median survival of 23.0 vs. 17.1 months for PLD alone (12). Thus, trabectedin + PLD appeared to be of particular benefit for those with partially platinum sensitive disease from second to further lines (12). Another post-hoc analysis of OVA-301 reported that in patients with relapsed ovarian cancer, trabectedin + PLD delays third-line chemotherapy and prolongs the platinum-free interval by about 2.5 months, and OS was also significantly prolonged in the partially platinum-sensitive disease subgroup (13). This adds additional support to the possibility that the enhanced survival benefits in the partially platinum-sensitive subset might be related to the ability of trabectedin to resensitize tumors to subsequent platinum rechallenge (7).

To further characterize the clinical efficacy of the trabectedin + PLD combination, we carried out a retrospective analysis involving 11 cases of recurrent ovarian tumor and partial platinum sensitivity.

\section{Materials and methods}

Patients and eligible criteria. A retrospective analysis was carried out at the Oncological Pharmacology Department at the University Hospital of Florence involving 11 patients with a diagnosis of ovarian epithelial tumor. All patients had previously undergone 1 cycle of platinum-based (carboplatin-taxol) chemo- therapy, without the addition of bevacizumab, from October 2011 to July 2014. All patients underwent chemotherapy with trabectedin and PLD, after a PFI of 6-12 months. An ECOG PS $\leq 2$ was required before initiating therapy as well as the following laboratory parameters: ( $\mathrm{Hb} \geq 9 \mathrm{~g} / \mathrm{dl}$, neutrophils $\geq 1.5 \times 10^{9} / 1$, platelets $\geq 100 \times 10^{9} / 1$, creatinine $<1.5 \mathrm{mg} / \mathrm{dl}$; bilirubin $\leq$ upper normal limit (ULN); ALT, AST, alkaline phosphatase, and creatinine phosphatase $\leq 2.5$ times ULN, albumin $\geq 25 \mathrm{~g} / \mathrm{l}$, and alkaline phosphatase $\leq 2.5$ times ULN.

Treatment and assessment. Trabectedin was administered every 3 weeks (i.v. $1.1 \mathrm{mg} / \mathrm{m}^{2}$ over $3 \mathrm{~h}$ ) immediately after the administration of PLD $\left(30 \mathrm{mg} / \mathrm{m}^{2}\right)$. All patients were premedicated with corticosteroids: Dexamethasone $20 \mathrm{mg}$ i.v. (prior to chemotherapy as an antiemetic), oral prednisone (10 mg BID starting the day before chemotherapy), followed by 1 day of $5 \mathrm{mg}$ BID (to avoid liver and hematologic toxicity due to trabectedin). Response to therapy was assessed by imaging (ultrasound and CT) every 3 cycles and by serum CA 125 levels at the beginning of each cycle. Patients with negative disease status underwent clinical and instrumental follow-up (ultrasound, CT, tumor markers, PET as needed) every 3 months. Recist 1.1 criteria were used for evaluation of response. The PFI was calculated from the last administration of chemotherapy. OS was calculated as the time from surgical intervention to death or last follow-up visit.

Adverse events. Adverse events were evaluated by clinical and laboratory assessment according to severity. In case of toxicity, the dose of trabectedin was reduced in 2 steps (1st step, $0.9 \mathrm{mg} / \mathrm{m}^{2}$ trabectedin and $25 \mathrm{mg} / \mathrm{m}^{2}$ PLD; 2nd step, $0.75 \mathrm{mg} / \mathrm{m}^{2}$ trabectedin and $\left.20 \mathrm{mg} / \mathrm{m}^{2} \mathrm{PLD}\right)$.

Statistical analysis. Statistical analysis was performed to evaluate objective response and PFS. PFS was expressed as median and interquartile range. Descriptive statistics were reported in terms of absolute frequencies and percentages for the qualitative data. PFS and OS were estimated according to the Kaplan-Meier method. The PFS and OS analyses were defined as the time interval from the last administration of trabectedin + PLD to the earliest date of disease progression or death for PFS, whereas OS was defined as the time from surgical intervention to death or last follow-up visit. The results were expressed as median values and mean values, with $95 \%$ confidence intervals (CIs).

\section{Results}

Patient cohort. A total of 11 patients, with median age of 60 years (range, $45-75$ years), with ovarian tumors were enrolled (Table I). All patients underwent debulking surgery (total hysterectomy, bilateral annessiectomy, omentectomy, appendicectomy and removal of peritoneal implants, when possible). In 9 patients, this was done at the beginning of clinical history, in 2 patients neoadjuvant chemotherapy was necessary (in 1 case for pelvic infiltration, in other case for pulmonary metastases) with subsequent interval surgery. Histological examination showed the following histotypes: 7 serous carcinomas, 1 endometrial carcinomas, 2 undifferentiated carcinomas, and 1 mucinous carcinoma. The degree of differentiation was low (G3) in 
Table I. Baseline characteristics of the patient cohort $(n=11)$.

\begin{tabular}{ll}
\hline Characteristic & Total, $\mathrm{n}(\%)$ \\
\hline Median age, years (range) & $60(45-75)$ \\
Surgery & \\
Primary & $9(82)$ \\
Interval & $2(18)$ \\
Stage & \\
IIB & $1(9)$ \\
IIC & $1(9)$ \\
IIIB & $1(9)$ \\
IIIC & $4(37)$ \\
IV & $4(37)$ \\
Histological type & \\
Serous & $7(64)$ \\
Endometrial & $1(9)$ \\
Mucinous & $1(9)$ \\
Undifferentiated & $2(18)$ \\
Grading & \\
1 & $1(9)$ \\
2 &
\end{tabular}

Table II. Response in the patient cohort $(n=11)$.

\begin{tabular}{lc}
\hline Response & $\begin{array}{c}\text { Trabectedin plus } \\
\text { PLD, n (\%) }\end{array}$ \\
\hline Complete response & $3(27.3)$ \\
Partial response & $1(9.0)$ \\
Stable disease & $3(27.3)$ \\
Progressive disease & $4(36.4)$ \\
Objective response rate & $7(63.6)$ \\
Median progression-free survival, & $5.9(0.6 ; 11.2)$ \\
months (95\% CI) & $32.4(22.4 ; 42.6)$ \\
Mean overall survival ${ }^{\text {a }}$, months $(95 \%$ CI) & \\
\hline${ }^{2}$ Median overall survival could not be calculated as some patients \\
were still alive. CI, confidence interval; PLD, pegylated liposomal \\
doxorubicin.
\end{tabular}

10 patients, while 1 patient had an intermediate degree of differentiation (G2). Regarding pathological stage: 2 cases were stage II, 7 cases stage III, and 2 cases stage IV. At the time of diagnosis, CA 125 was elevated in 10 patients, while it was below the threshold value in 1 patient. All patients underwent
Table III. Adverse events $(\mathrm{n}=11)$.

\begin{tabular}{lccc}
\hline & \multicolumn{3}{c}{ Trabectedin plus PLD, n (\%) } \\
\cline { 2 - 4 } Adverse event & Grade 1 & Grade 2 & Grade 3 \\
\hline Neutropenia & $4(36.3)$ & $3(27.2)$ & $2(18.2)$ \\
Anemia & $5(45.4)$ & $3(27.2)$ & 0 \\
Thrombocytopenia & $3(27.2)$ & $1(0.1)$ & 0 \\
Cutaneous toxicity & $2(18.2)$ & $2(18.2)$ & $2(18.2)$ \\
Nausea/vomiting & $2(18.2)$ & $2(18.2)$ & 0 \\
Hepatotoxicity & $4(36.3)$ & $3(27.2)$ & 0 \\
Asthenia & $3(27.2)$ & $1(0.1)$ & 0 \\
Blood creatine & $2(18.2)$ & 0 & 0 \\
phosphokinase increased & & & \\
\hline
\end{tabular}

No Grade 4 events were observed.PLD, pegylated liposomal doxorubicin

first-line chemotherapy with carboplatin and taxol for a total number of cycles ranging from 6 to 9 .

The 2 patients undergoing neoadjuvant chemotherapy performed both 4 pre-intervention chemotherapy cycles and 4 cycles of the same pattern after interval surgery. The recurrences, all with a free interval between 6 and 12 months, were found in 4 patients at lymph node sites, ( 1 of these patients experienced a thyroid metastasis), and in 7 patients at peritoneal sites (peritoneal carcinosis). At the time of recurrence, CA 125 was positive in 7 of 11 patients.

All 11 patients had undergone trabectedin and PLD as second-line therapy. A median of 6 cycles of chemotherapy were administered (range 1-12). The 7 patients who underwent a third line were treated with carboplatin and gemcitabine. At that time, a genetic test for the BRCA 1-2 gene was not routinely performed, and so data are available for only 2 of the 3 patients who are still alive at the time of writing. One patient has a BRCA1 mutation, 1 wild-type, and for the third genetic response is pending.

Efficacy. In the 11 patients, 4 showed complete response, 3 achieved stable disease, and 4 had progression of disease. Accordingly, $63.6 \%$ of patients responded to therapy (complete or partial response or stable disease) (Table II). Mean OS was 32.42 months (95\% CI 22.4; 42.6) (Fig. 1). The median PFS was 5.9 months (95\% CI 0.6; 11.2) (Table II, Fig. 2). Of the 4 patients who progressed during therapy, 2 patients had a serous histology, 1 patient had mucinous histology (the only patient with stage II), and 1 patient had endometrial carcinoma. The complete responses were in patients with serous $(n=2)$ and undifferentiated carcinoma $(n=1)$, and the partial response was in a patient with serious disease. Of the 4 patients with progression, 1 died after a single cycle of therapy. The 7 patients who had relapsed all underwent a third line with carboplatin and gemcitabine. Of these patients, 2 had a complete response, 2 a partial response, 2 were stationary, and 1 had progression after the third cycle of therapy. The 2 patients who had a complete response had a disease-free interval of 6 and 8 months, respectively. Median OS for these patients was 45 months (range 7-75 months). 
Tolerability. Regarding treatment-related toxicity, grade 3 cutaneous toxicity was seen in $18.2 \%$ of patients, despite prophylaxis with vitamin B6 (300 mg/day orally). Trabectedin in combination with PLD was well tolerated in terms of gastrointestinal and hematological toxicity. Grade 3 neutropenia was observed in $18.2 \%$ of patients, although there was no need for treatment delay, discontinuation, or dose adjustments, but only growth factors to treat neutropenia (Table III).

\section{Discussion}

In the present retrospective study, median PFS was 9 months in the 11 patients with recurrent ovarian cancer and a PFI of 6-12 months receiving the combination of trabectedin + PLD. This is in good general agreement with data from the OVA-301 trial where patients with a PFI of 6-12 months receiving the same combination were found to have a median PFS of 7.4 months (13). Our data further reinforce the utility of trabectedin + PLD in patients with relapsed ovarian cancer, and especially in those with partial platinum sensitivity. Considering the available data, trabectedin and PLD appears to be a valid therapeutic option for second and further-line therapy in patients experiencing relapse after 6-12 months following first-line therapy with carboplatin-paclitaxel-bevacizumab.

In the final analysis of OVA-301, median OS in the overall population for the trabectedin + PLD and PLD arms was 22.2 and 18.9 months, respectively (HR, $0.86 ; \mathrm{P}=0.0835)$ (14). Moreover, in an exploratory analysis, the subset of patients with a PFI of 6-12 months had the largest difference in OS (22.4 vs. 16.4 months with $\mathrm{HR}=0.64 ; \mathrm{P}=0.0027)$. As noted previously, the positive trend toward a survival advantage with the trabectedin + PLD compared to PLD alone in the PFI 6- to 12-month subgroup of patients does not appear to be related to the effects of subsequent therapies received by the patients after discontinuing study medication (12). In fact, about the same proportion of patients in each group received subsequent therapy, and patients treated with trabectedin + PLD actually had a slightly lower proportion of additional platinum-based treatment than did patients treated with PLD alone. In OVA-301, the administration of additional platinum-containing lines of therapy was significantly delayed for patients receiving trabectedin + PLD, which significantly prolonged survival starting from the initiation of subsequent platinum. Accordingly, the survival benefits with trabectedin + PLD compared to PLD alone in patients with partially platinum-sensitive disease is possibly due to extension of the PFI through resensitization of tumors to platinum by trabectedin $(7,15)$. In addition to an advantage in survival the increased interval before reinitiating therapy also provides the patient more time to recover from the adverse effects of previous platinum therapy.

Our results are also similar to those published in a recent real-life study in 17 patients treated with trabectedin alone or combined with PLD (16). In that study, median PFS was 6.7 months, while median OS was 17.6 months. Positive experience in a smaller real-life setting was also reported in 6 patients; the authors further documented a heavily-treated patient with advanced recurrent ovarian cancer treated with trabectedin + PLD at fourth-line who was later rechallenged at seventh-line, with treatment continuing until disease progression (17). The findings are also in broad agreement with

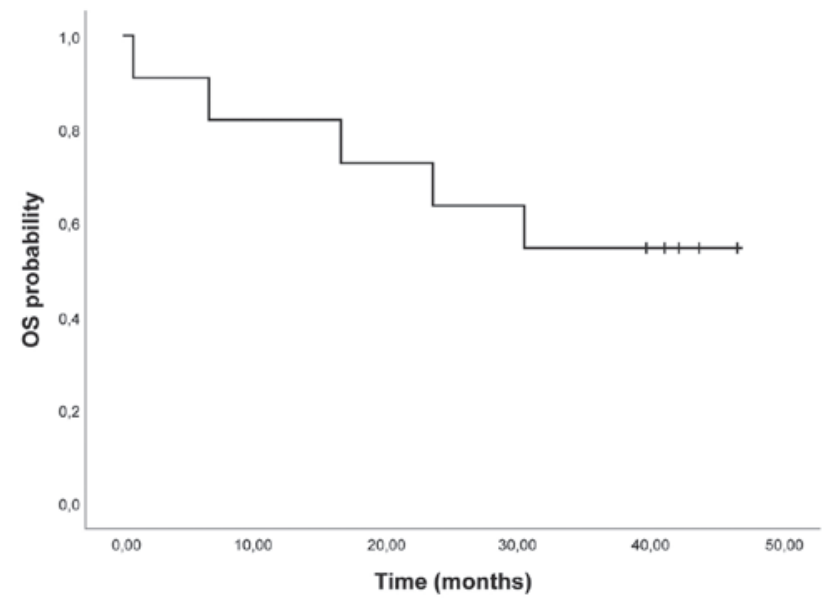

Figure 1. Kaplan-Meier estimate of OS ( $\mathrm{n}=11)$. There were 5 patients with events; 6 patients were censored. OS, overall survival.

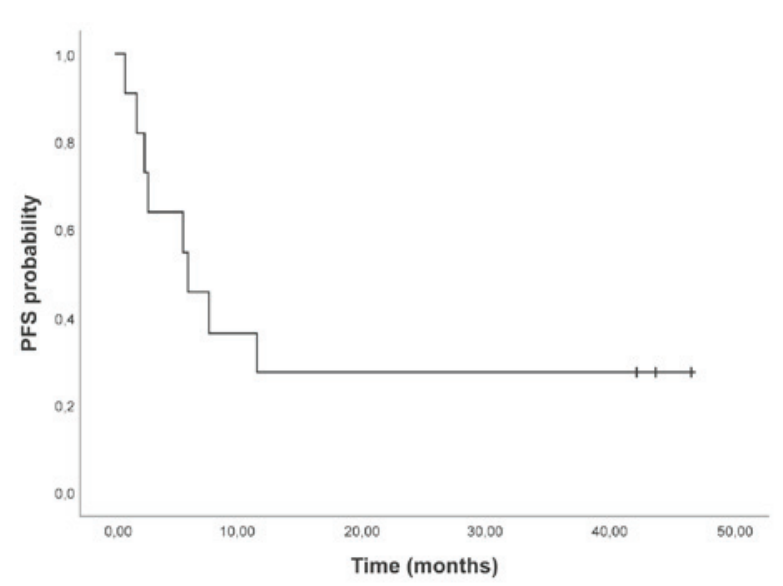

Figure 2. Kaplan-Meier estimate of PFS ( $\mathrm{n}=11)$. There were 8 patients with events; 3 patients were censored. PFS, progression-free survival.

a small retrospective analysis of heavily pretreated patients with platinum-sensitive ovarian cancer in which the ORR was $32.4 \%$ with median OS of 16.3 months; most responses were in (9 of 11) patients with partially platinum-sensitive disease (18).

Regarding tolerability, there were no unexpected safety signals in the present cohort of patients. In the OVA-301 trial, the safety profile in the subgroup of patients with a PFI of 6-12 months was not different from that in the overall population, even if compared with single-agent PLD, trabectedin + PLD was associated with a higher incidence of transient neutropenia and transaminase elevations (12). Moreover, the addition of trabectedin to PLD did not lead to a decrease in overall health status as assessed by patient reported outcomes. Grade 3 neutropenia was observed in $\leq 20 \%$ of patients, and treatment was neither delayed nor discontinued, and no dose adjustments were needed. This finding is consistent with the real-life study by Moriceau where the most frequent grade 3-4 toxicities were neutropenia (24\%) and nausea/vomiting (24\%) (16). Thus, trabectedin combined with PLD appears to be both effective and well tolerated in a real-life setting of women with recurrent ovarian cancer, and the toxicity of this combination is predictable and manageable. This is an important aspect as there is a high unmet need for new and tolerable therapies. 
A good tolerability profile for trabectedin was also reported in the large case study of Araki comparing trabectedin with the best supportive care in patients with advanced translocation-related sarcoma after failure of standard chemotherapy (19) and in a recent case report of our group where the patient continued the treatment with trabectedin for a total of 30 months without significant toxicity (20).

Of note, the trabectedin + PLD combination has been recommended by ESMO in patients with relapsed partially platinum-sensitive ovarian cancer (21). While the limitations of the present study include its retrospective design and small number of patients, our results nonetheless reinforce the validity of trabectedin + PLD in patients with relapsed ovarian cancer and partial platinum sensitivity.

\section{Acknowledgements}

Not applicable.

\section{Funding}

The present study was supported by PharmaMar, S.A. (Madrid, Spain).

\section{Availability of data and materials}

The datasets used and/or analyzed during the current study are available from the corresponding author on reasonable request.

\section{Authors' contributions}

KT, LV and AV conceived and designed the study. VR and LV collected the patient data. SN, KT, LV, VR and AV participated in data analysis and interpretation. LV, AV, GA, TM and EM drafted and revised the manuscript critically for important intellectual content. All authors read and approved the final manuscript.

\section{Ethics approval and consent to participate}

Written informed consent was obtained at the time of original data collection.

\section{Patient consent for publication}

Not applicable.

\section{Competing interests}

The authors declare that they have no competing interests.

\section{References}

1. Colombo N: Optimizing treatment of the partially platinum-sensitive ovarian cancer patient. Future Oncol 9 (12 Suppl): S19-S23, 2013

2. Friedlander M,TrimbleE,Tinker A, Alberts D, Avall-LundqvistE, Brady M, Harter P, Pignata S, Pujade-Lauraine E, Sehouli J, et al: Clinical trials in recurrent ovarian cancer. Int J Gynecol Cancer 21: 771-775, 2011

3. González-Martín A: Update on relapsed ovarian cancer treatment: From new consensus to daily clinical practice. Future Oncol 13 (23s): 3-9, 2017
4. Gore ME, Fryatt I, Wiltshaw E and Dawson T: Treatment of relapsed carcinoma of the ovary with cisplatin or carboplatin following initial treatment with these compounds. Gynecol Oncol 36: 207-211, 1990.

5. Markman M, Rothman R, Hakes T, Reichman B, Hoskins W, Rubin S, Jones W, Almadrones L and LewisJL Jr: Second-line platinum therapy in patients with ovarian cancer previously treated with cisplatin. J Clin Oncol 9: 389-393, 1991.

6. López-Guerrero JA, Romero I and Poveda A: Trabectedin therapy as an emerging treatment strategy for recurrent platinum-sensitive ovarian cancer. Chin J Cancer 34: 41-49, 2015.

7. González-Martín A and du Bois A: Factors to consider and questions to ask in the management of recurrent ovarian cancer: A focus on the role of trabectedin + pegylated liposomal doxorubicin. Expert Rev Anticancer Ther 16 (Sup1): S3-S10, 2016

8. D'Incalci $M$ and Galmarini CM: A review of trabectedin (ET-743): A unique mechanism of action. Mol Cancer Ther 9: 2157-2163, 2010.

9. Guirouilh-Barbat J, Redon C and Pommier Y: Transcriptioncoupled DNA double-strand breaks are mediated via the nucleotide excision repair and the Mre11-Rad50-Nbs1 complex. Mol Biol Cell 19: 3969-3981, 2008.

10. Allavena P, Signorelli M, Chieppa M, Erba E, Bianchi G, Marchesi F, Olimpio CO, Bonardi C, Garbi A, Lissoni A, et al: Anti-inflammatory properties of the novel antitumor agent yondelis (trabectedin): Inhibition of macrophage differentiation and cytokine production. Cancer Res 65: 2964-2971, 2005.

11. Monk BJ, Herzog TJ, Kaye SB, Krasner CN, Vermorken JB, Muggia FM, Pujade-Lauraine E, Lisyanskaya AS, Makhson AN, Rolski J, et al: Trabectedin plus pegylated liposomal Doxorubicin in recurrent ovarian cancer. J Clin Oncol 28: 3107-3114, 2010.

12. Poveda A, Vergote I, Tjulandin S, Kong B, Roy M, Chan S, Filipczyk-Cisarz E, Hagberg H, Kaye SB, Colombo N, et al: Trabectedin plus pegylated liposomal doxorubicin in relapsed ovarian cancer: Outcomes in the partially platinum-sensitive (platinum-free interval 6-12 months) subpopulation of OVA-301 phase III randomized trial. Ann Oncol 22: 39-48, 2011.

13. Kaye SB, Colombo N, Monk BJ, Tjulandin S, Kong B, Roy M, Chan S, Filipczyk-Cisarz E, Hagberg H, Vergote I, et al: Trabectedin plus pegylated liposomal doxorubicin in relapsed ovarian cancer delays third-line chemotherapy and prolongs the platinum-free interval. Ann Oncol 22: 49-58, 2011.

14. Monk BJ, Herzog TJ, Kaye SB, Krasner CN, Vermorken JB, Muggia FM, Pujade-Lauraine E, Park YC, Parekh TV and Poveda AM: Trabectedin plus pegylated liposomal doxorubicin (PLD) versus PLD in recurrent ovarian cancer: Overall survival analysis. Eur J Cancer 48: 2361-2368, 2012.

15. Colombo N: Optimising the treatment of the partially platinum-sensitive relapsed ovarian cancer patient. EJC Suppl 12: 7-12, 2014.

16. Moriceau G, Rivoirard R, Méry B, Vallard A, Pacaut C, Trone JC, Espenel S, Bosacki C, Jacquin JP and Magné N: Real-world outcomes of combination chemotherapy with trabectedin plus pegylated liposomal doxorubicin in patients with recurrent ovarian cancer: a single-center experience. Chemotherapy 61: 122-126, 2016.

17. Tahir S: Real-life experience using trabectedin plus pegylated liposomal doxorubicin combination to treat patients with relapsed ovarian cancer. EJC Suppl 12: 17-20, 2014.

18. Nicoletto MO, Baldoni A, Casarin A, Randon G, Nardin M, Baretta Z, Lardelli P, Nieto A, Alfaro V, Rigamonti C and Conte PF: Trabectedin plus pegylated liposomal doxorubicin: Retrospective analysis in heavily pretreated platinum-sensitive ovarian cancer. Tumori 101: 506-510, 2015.

19. Araki N, Takahashi S, Sugiura H,Ueda T, Yonemoto T, Takahashi M, Morioka H, Hiraga H, Hiruma T, Kunisada T, et al: Retrospective inter- and intra-patient evaluation of trabectedin after best supportive care for patients with advanced translocation-related sarcoma after failure of standard chemotherapy. Eur J Cancer 56: 122-130, 2016.

20. Tavella K, Villanucci A, Vannini L, Lavacchi D, Montelatici S, Amunni G and Mazzei T: Stable disease in a patient with metastatic leiomyosarcoma treated with trabectedin. Anticancer Drugs 28: 465-468, 2017.

21. Ledermann JA, Raja FA, Fotopoulou C, Gonzalez-Martin A, Colombo N and Sessa C; ESMO, Guidelines Working Group: Newly diagnosed and relapsed epithelial ovarian carcinoma: ESMO Clinical Practice Guidelines for diagnosis, treatment and follow-up. Ann Oncol 24 (Suppl 6): vi24-vi32, 2013. 